\title{
Pain Free Efficacy of Sumatriptan in the Early Treatment of Migraine
}

\author{
Susan E. Jelinski, Werner J. Becker, Suzanne N. Christie, Faiz F. Ahmad, \\ William Pryse-Phillips, Scott D. Simpson
}

\begin{abstract}
Background: There is evidence that headache response rates may be higher if triptans are used early when a migraine attack is still mild, as compared to when it is treated after pain has reached moderate or severe intensity. Methods: In this randomized, double blind, placebo controlled, parallel group clinical trial, 361 patients took either placebo, sumatriptan $50 \mathrm{mg}$, or sumatriptan $100 \mathrm{mg}$ in a single attack study. The primary outcome measure was pain-free status at two hours. Results: In the intention to treat group, two hour pain free rates were $16 \%, 40 \%$, and $50 \%$ in the placebo group, sumatriptan $50 \mathrm{mg}$ group, and the sumatriptan $100 \mathrm{mg}$ group respectively $(\mathrm{p}<0.001$, active treatment groups vs. placebo). Conclusions: Both sumatriptan $50 \mathrm{mg}$ and $100 \mathrm{mg}$ were significantly superior to placebo for the pain-free end point at two hours. The pain-free response rates in this trial where sumatriptan was taken while the headache was still mild were generally higher than in older clinical trials where headache was treated after reaching a moderate or severe intensity.
\end{abstract}

RÉSUMÉ: Absence de douleur comme mesure de l'efficacité du sumatriptan pour le traitement précoce de la migraine. Contexte: Le taux de réponse serait plus élevé si les triptans sont utilisés tôt au cours d'une crise de migraine alors que les symptômes sont légers plutôt que quand la douleur est devenue modérée ou sévère. Méthodes: Il s'agit d'une étude de pharmacologie clinique randomisée, à double insu, avec placebo et groupes parallèles. 361 patients ont pris soit le placebo, le sumatriptan $50 \mathrm{mg}$ ou le sumatriptan $100 \mathrm{mg}$ au cours d'une seule crise migraineuse. Le critère d'évaluation primaire était l'absence de douleur 2 heures après la prise du médicament. Résultats: À l'analyse selon l'intention de traitement, le taux de patients sans douleur était de $16 \%, 40 \%$ et $50 \%$ pour le groupe placebo, le groupe sumatriptan $50 \mathrm{mg}$ et le groupe sumatriptan $100 \mathrm{mg}$ respectivement (groupes traitement actif versus placebo, p > 0,001). Conclusions: Le sumatriptan $50 \mathrm{mg}$ et $100 \mathrm{mg}$ étaient significativement supérieurs au placebo quant au critère d'évaluation primaire, soit l'absence de douleur 2 heures après la prise du médicament. Le taux de réponse, soit l'absence de douleur, dans cette étude où le sumatriptan était pris alors que la céphalée était encore légère, était généralement plus élevé que dans d'autres essais cliniques où la céphalée était traitée alors qu'elle était modérée ou sévère.

Can. J. Neurol. Sci. 2006; 33: 73-79

Migraine is a common neurological disorder that is characterized by episodic headaches associated with symptoms such as nausea, visual disturbances, photophobia, and phonophobia. In Canada, approximately $7 \%$ of adult males and $22 \%$ of adult females suffer from migraine. ${ }^{1}$ Migraine symptoms result in a considerable burden with regard to health care utilization, health care costs, and work/productivity loss due to disability and decreased functional status. ${ }^{2-6}$

Clinical trials have demonstrated that sumatriptan (Imitrex ${ }^{\circledR}$, GlaxoSmithKline) is effective in relieving migraine pain. These studies have established the efficacy of sumatriptan among patients who treated their migraines at a point in which their headache pain was classified as moderate or severe. ${ }^{7-11}$ However, from a neurobiological perspective, it is plausible that pharmacotherapy initiated at an earlier stage of migraine pain development may be more efficacious in eliminating headaches.

From the Department of Clinical Neurosciences, University of Calgary (SEJ, WJB), Calgary, AB; Ottawa Headache Centre (SNC), Ottawa, ON; GlaxoSmithKline (FFA, WPP, SDS), Mississauga, ON; Faculty of Medicine, Memorial University of Newfoundland (WPP), St. John's, NL, Canada.

ReCEIVEd APRIL 26, 2005. ACCEPTEd IN FINAL FORM SEPTEMBER 24, 2005. Reprint requests to: Werner J. Becker, Department of Clinical Neurosciences, Foothills Medical Centre, 12th Floor, Neurology, 1403-29th Street NW, Calgary, Alberta, T2N 2T9, Canada. 
Migraine pain is thought to arise from cerebrovascular nociceptors which transmit through peripheral sensory nerves to central sensory neurons. During a migraine attack these central sensory neurons may become sensitized, ('central sensitization') resulting in further pain progression. ${ }^{12}$ This hypothesized pathophysiologic mechanism is a valid rationale for the early treatment of migraine pain.

A growing body of clinical evidence supports the neurobiological evidence that early treatment may be more effective in relieving migraine pain than treatment at a later stage in the evolution of the migraine attack. A retrospective subgroup analysis performed on protocol violators in a migraine trial suggested that sumatriptan is most effective in relieving migraine pain when treatment is initiated at an earlier, mild stage of migraine pain. ${ }^{13}$ Recent clinical trials have demonstrated similar results with sumatriptan ${ }^{14,15}$ and other triptans. ${ }^{16,17}$

The present study was conducted in order to augment the evidence supporting the early treatment of migraine pain with sumatriptan. The objective of this randomized, double-blinded, placebo-controlled trial was to determine the efficacy of $50 \mathrm{mg}$ and $100 \mathrm{mg}$ sumatriptan compared with placebo in providing complete pain relief at two hours after treatment when treatment was initiated at a mild pain stage within two hours of migraine attack onset.

\section{Methods}

\section{Study Design}

This was a multi-centre, randomized, double-blind, doubledummy, placebo controlled, parallel-group clinical trial, conducted at 25 sites in Canada during 2001 and 2002 (protocol \# sum40291). The research protocol was submitted and approved by the relevant ethics board at each participating centre.

\section{Patient Recruitment}

Male and female migraine patients between the ages of 18 and 65 years were invited to participate in the clinical trial. Patients were recruited from the practices of 26 participating physicians in Canada. Participants included neurologists who specialized in headache, a number of community neurologists, and several family physicians with a special interest and large experience in headache management. In order to meet inclusion criteria, subjects had to meet the International Headache Society diagnostic criteria for migraine with or without aura, ${ }^{18}$ have had one to six migraine attacks per month in the two months prior to screening for participation in the clinical trial, and typically had to experience moderate to severe migraine pain preceded by a mild pain phase. These clinical features were confirmed before study entry by a detailed clinical history taken from the patient. Patients had to be capable of reading, comprehending, and completing subject questionnaires, and willing to provide informed, written consent to participate in the study. Exclusion criteria included confirmed or suspected ischemic heart disease, cardiac arrhythmias, hypertension, impaired hepatic or renal function, and a history of cerebrovascular disease, congenital heart disease, ischemic abdominal syndromes, peripheral vascular disease, Raynaud syndrome, or epilepsy. Patients diagnosed with basilar migraine, hemiplegic migraine, cluster headache, or who showed evidence of a rebound headache pattern caused by ergotamine or analgesic overuse in the three months prior to enrollment were excluded. Patients taking monoamine oxidase inhibitor drugs were not enrolled. Pregnant or breast-feeding women and those not using adequate contraception were also excluded from the trial.

Those patients meeting inclusion and exclusion criteria, and who provided informed, written consent, were randomized to one of three groups; $50 \mathrm{mg}$ sumatriptan, $100 \mathrm{mg}$ sumatriptan, or placebo. Patients were assigned treatment numbers according to a randomization schedule generated by the Medical Data Sciences Department of Glaxo Wellcome Canada Inc. The ratio of treatment assignment was 1:1:1 with a block size of 6 . Treatment group assignment was unknown to patients and investigators.

\section{Study Procedures}

At the initial screening visit, data regarding medical and migraine history, demographics, and medication history were collected for each patient. The Headache Impact Test (HIT-6) was used to evaluate disability experienced by the migraine patients because of their headaches. ${ }^{19}$ Patients were instructed to treat their next migraine attack within two hours of the first sign of migraine pain, while the pain was still considered to be 1 (mild) on a four point scale $(0=$ no pain, $1=$ mild pain, $2=$ moderate pain, $3=$ severe pain). If the patient experienced moderate to severe migraine pain at the onset of the attack, they were instructed not to treat that migraine with the study medication. Subjects were permitted to treat headache recurrence with a second dose of study medication two to 24 hours after the first dose of the study medication was taken. They were instructed to withhold consumption of any analgesics, anti-emetics, or other acute migraine medications within six hours prior to and two hours after the first dose of the study medication. As well, use of ergotamine, ergot-type medications, or other $5 \mathrm{HT}_{1}$ agonists was restricted for 24 hours before and after study medication use. Patients were permitted to continue their use of prophylactic medications (with the exception of methysergide) during the study, as long as they had been on a stable dose starting at least one month before study entry.

A headache diary and questionnaire were given to each patient with instructions to complete both data forms following their treated migraine attack. The diary was used to record details about the patients' headaches, such as headache severity, headache recurrence, use of additional medications, and presence of migraine symptoms (nausea, vomiting, photophobia, phonophobia). Patients were instructed to record the severity of their headache immediately before taking study medication, and at $30 \mathrm{~min}$, one, two, four, and 24 hours after taking study medication. Headache severity was graded on a four point scale $(0=$ no pain, $1=$ mild pain, $2=$ moderate pain, $3=$ severe pain). Those patients who experienced complete pain relief within two hours following the first dose of the study medication were asked to record whether their headache recurred between two and 24 hours after taking study medication.

\section{Data Analysis}

Both intention-to-treat (ITT) and per-protocol (PP) analyses were performed. The authors had full access to the data from the 


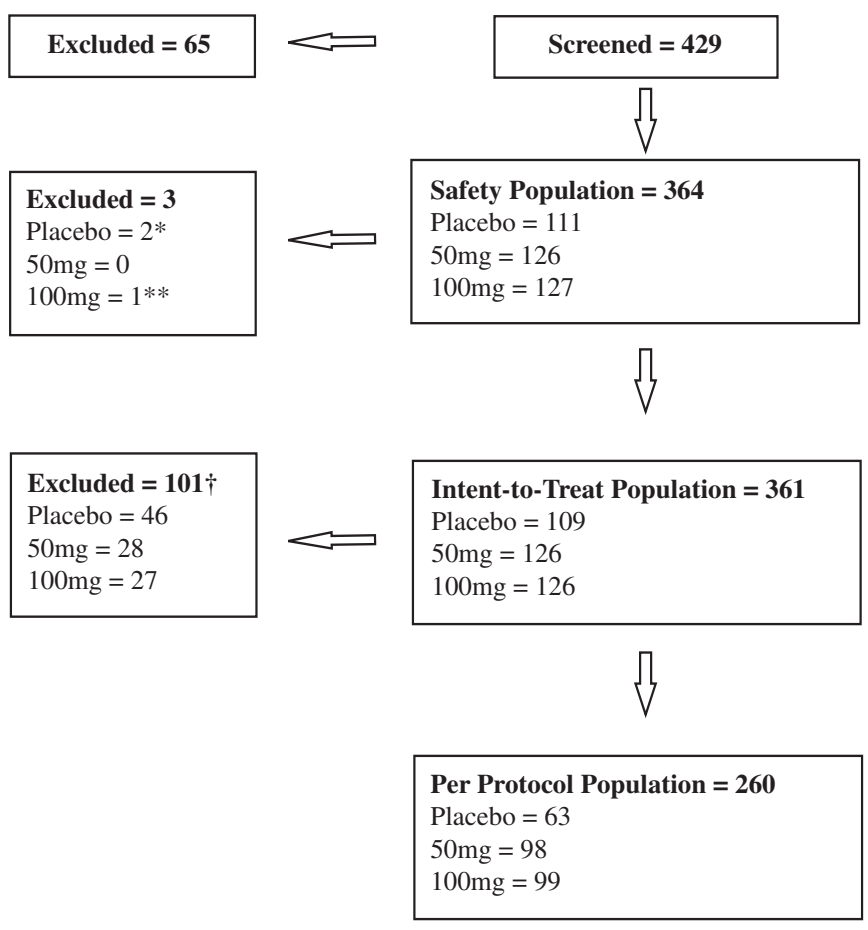

Figure: Patient Disposition. *One subject withdrew from the study and one was lost to follow-up. **One was lost to follow-up. †Half of the excluded ITT patients (51\%) took restricted medications 24 hours before or after consuming the study medication; $15 \%$ did not take study medication within two hours of migraine onset.

clinical trial. The ITT population included those subjects who provided an evaluation of the treatment with the randomized study medication. The PP population comprised those patients in the ITT population who complied with all aspects of the study treatment protocol. These included use of the randomized medication to treat the migraine pain at a mild stage and within two hours of migraine pain onset; provision of an evaluation of their randomized treatment; avoidance of the use of ergotamine or ergot-type medications 24 hours before or after using the study medication; and no exposure to any other type of 5- $\mathrm{HT}_{1}$ agonists within 24 hours of dosing with the randomized treatment.

The Cochran Mantel-Haenszel test, controlling for study centre, was used to test for statistically significant differences between both $50 \mathrm{mg}$ and $100 \mathrm{mg}$ sumatriptan and placebo groups in clinical efficacy as measured by the percentage of subjects who achieved complete relief of their headache pain at various time points following administration of the study medication. The primary endpoint of the study was the percentage of subjects experiencing complete relief of migraine pain two hours after treatment with sumatriptan $50 \mathrm{mg}$ as compared to placebo.

\section{RESULTS}

The disposition of subjects and the groups of patients used for statistical analyses are shown in the Figure. Three hundred and sixty-four patients were randomized to the three study groups, of whom 361 treated a migraine attack (Intention to Treat population, ITT). Of these 361 subjects, 101 (28\%) violated the study protocol. Twenty-eight percent (28\%) of placebo patients, $11 \%$ in the $50 \mathrm{mg}$ sumatriptan group, and $8 \%$ in the $100 \mathrm{mg}$ sumatriptan group violated protocol by taking restricted medications within 24 hours before or 24 hours after taking study medication.

Table 1 displays the study patients' characteristics and demographics at baseline. Patients were predominately white and female, with a mean age of approximately 40 years. The mean HIT-6 scores for all groups of patients were above 70 , indicating that the study patients were experiencing disabling headache pain that severely impacted their lives. There were no substantial differences between the three treatment groups with regard to age, gender, race, use of oral contraceptives among the female subjects, migraine history, or mean HIT-6 scores.

The pain-free rates for the three treatment groups in the ITT and the PP populations are shown in Table 2. For both the $50 \mathrm{mg}$ and $100 \mathrm{mg}$ groups, sumatriptan was superior to placebo in achieving a pain-free state at one hour after study medication and onward up to 24 hours. At two hours, pain-freedom was achieved by $40 \%$ in the $50 \mathrm{mg}$ sumatriptan group and $50 \%$ in the $100 \mathrm{mg}$ sumatriptan group (ITT population). These results were statistically significant compared to the $16 \%$ pain-free response rate in the placebo group (both $\mathrm{p}<0.001$ ). The results for the PP populations were similar, with pain free response rates at two hours of $49 \%$ and $57 \%$ for the sumatriptan $50 \mathrm{mg}$ group and the $100 \mathrm{mg}$ group respectively (placebo response $21 \%, \mathrm{p}<0.001$ for both sumatriptan groups vs. placebo). For the sumatriptan 100 $\mathrm{mg}$ group (ITT analysis), pain-free response rates at 30 minutes were also significantly different from placebo at the $\mathrm{p}<0.01$ level (placebo 4\%, sumatriptan 8\%).

Table 1: Patient Characteristics and Demographics (ITT Population*)

\begin{tabular}{|c|c|c|c|}
\hline $\begin{array}{l}\text { Number of } \\
\text { Patients }\end{array}$ & $\begin{array}{l}\text { Placebo } \\
n=109\end{array}$ & $\begin{array}{l}50 \mathrm{mg} \\
\mathrm{n}=126\end{array}$ & $\begin{array}{l}100 \mathrm{mg} \\
\mathrm{n}=126\end{array}$ \\
\hline Age (mean) & $40.7 \pm 9.8$ & $39.8 \pm 9.7$ & $39.8 \pm 11.4$ \\
\hline Sex (female) & $91(83 \%)$ & $110(87 \%)$ & $108(86 \%)$ \\
\hline Race (white) & $100(92 \%)$ & $120(95 \%)$ & $121(96 \%)$ \\
\hline $\begin{array}{l}\text { Use of Oral } \\
\text { Contraceptives }\end{array}$ & $16(18 \%))$ & $22(20 \%)$ & $24(19 \%)$ \\
\hline \multicolumn{4}{|l|}{ Migraine History } \\
\hline Without Aura & $73(67 \%)$ & $80(63 \%)$ & $89(71 \%)$ \\
\hline With Aura & $11(10 \%)$ & $12(10 \%)$ & $9(7 \%)$ \\
\hline $\begin{array}{l}\text { With and } \\
\text { Without Aura }\end{array}$ & $25(23 \%)$ & $34(27 \%)$ & $28(22 \%)$ \\
\hline HIT-6 Score (mean) & $75.8 \pm 22.0$ & $73.9 \pm 18.0$ & $70.9 \pm 14.4$ \\
\hline
\end{tabular}

*ITT $=$ Intention To Treat 
Table 2: Pain-Free Rates for Sumatriptan $50 \mathrm{mg}$ and $100 \mathrm{mg}$ Compared with Placebo

\begin{tabular}{|c|c|c|c|c|c|}
\hline \multirow[b]{2}{*}{ Time After } & \multicolumn{3}{|c|}{ ITT Population* } & \multicolumn{2}{|c|}{ P Value } \\
\hline & Placebo & $50 \mathrm{mg}$ & $100 \mathrm{mg}$ & $50 \mathrm{mg}$ vs & $100 \mathrm{mg} \mathrm{vs}$ \\
\hline First Dose & $\mathrm{n}=109$ & $\mathrm{n}=126$ & $\mathrm{n}=126$ & placebo & placebo \\
\hline $30 \mathrm{~min}$ & $1(<1 \%)$ & $5(4 \%)$ & $10(8 \%)$ & 0.099 & 0.009 \\
\hline $1 \mathrm{hr}$ & $8(7 \%)$ & $30(24 \%)$ & $30(24 \%)$ & $<0.001$ & $<0.001$ \\
\hline $2 \mathrm{hr}$ & $17(16 \%)$ & $51(40 \%)$ & $63(50 \%)$ & $<0.001$ & $<0.001$ \\
\hline $4 \mathrm{hr}$ & $19(17 \%)$ & $63(50 \%)$ & $71(56 \%)$ & $<0.001$ & $<0.001$ \\
\hline \multirow[t]{2}{*}{$24 \mathrm{hrs}$} & $16(15 \%)$ & $47(37 \%)$ & $57(45 \%)$ & $<0.001$ & $<0.001$ \\
\hline & \multicolumn{3}{|c|}{ PP Population** } & \multicolumn{2}{|c|}{ P Value } \\
\hline Time After & Placebo & $50 \mathrm{mg}$ & $100 \mathrm{mg}$ & $50 \mathrm{mg} \mathrm{vs}$ & $100 \mathrm{mg} \mathrm{vs}$ \\
\hline First Dose & $\mathrm{n}=63$ & $\mathrm{n}=98$ & $\mathrm{n}=99$ & placebo & placebo \\
\hline $30 \mathrm{~min}$ & $0(0 \%)$ & $5(5 \%)$ & $9(9 \%)$ & 0.064 & 0.019 \\
\hline $1 \mathrm{hr}$ & $6(10 \%)$ & $29(30 \%)$ & $26(26 \%)$ & 0.006 & 0.006 \\
\hline $2 \mathrm{hr}$ & $13(21 \%)$ & $48(49 \%)$ & $56(57 \%)$ & 0.001 & $<0.001$ \\
\hline $4 \mathrm{hr}$ & $14(22 \%)$ & $56(57 \%)$ & $60(61 \%)$ & $<0.001$ & $<0.001$ \\
\hline $24 \mathrm{hrs}$ & $14(22 \%)$ & $42(43 \%)$ & $51(52 \%)$ & 0.027 & $<0.001$ \\
\hline
\end{tabular}

*ITT = Intention To Treat; ** Per Protocol

Table 3 demonstrates the proportions of patients who maintained a pain-free response between two and 24 hours after taking study medication. In the ITT analysis, the percentage of subjects who sustained a pain-free response for both $50 \mathrm{mg}$ and $100 \mathrm{mg}$ sumatriptan groups (24\% and $27 \%$ ) was significantly higher than in the placebo group $(6 \%)$. The PP analysis provided similar results.

The proportions of patients with worsening of headache at two hours and four hours after consuming the study medication are presented in Table 4. For both the $50 \mathrm{mg}$ and $100 \mathrm{mg}$ sumatriptan groups, the percentage of patients who had worsening of their migraine pain was significantly less compared to placebo. After four hours, $25 \%$ of the $50 \mathrm{mg}$ sumatriptan group and $13 \%$ of the $100 \mathrm{mg}$ sumatriptan group experienced worsening of their migraine pain, compared to $46 \%$ of placebo patients (both $\mathrm{p}<0.001$, ITT population).

For subjects who were pain-free at two hours, some degree of headache returned between two and 24 hours after taking study medication in $47 \%$ of subjects in the placebo group, $39 \%$ in the sumatriptan $50 \mathrm{mg}$ group, and $44 \%$ in the sumatriptan $100 \mathrm{mg}$ group. Median time to return of migraine was 3.8 hours in the placebo group, 12.2 hours in the sumatriptan $50 \mathrm{mg}$ group, and 12.3 hours in the sumatriptan $100 \mathrm{mg}$ group.

Sumatriptan was generally well tolerated, and there were no serious adverse events in either sumatriptan group. Adverse events considered by the investigators to be drug related occurred in $3 \%, 17 \%$, and $22 \%$ in the placebo, sumatriptan 50 $\mathrm{mg}$, and sumatriptan $100 \mathrm{mg}$ groups respectively. In the sumatriptan $100 \mathrm{mg}$ group, which had numerically more adverse events than the other groups, paraesthesias, chest symptoms, and throat constriction were each reported by $3 \%$ of subjects.

At two hours after taking study medication, nausea was reported by $38 \%$ of patients in the placebo group, and by $26 \%$ of patients in each of the active treatment groups.

\section{Discussion}

The objectives of this randomized controlled trial included determination of the efficacy of sumatriptan $50 \mathrm{mg}$ and $100 \mathrm{mg}$ in the early treatment of migraine during the mild phase of migraine pain, compared to placebo. Both doses of sumatriptan provided statistically significant superior efficacy as compared to placebo in providing complete pain relief at one, two, and four hours after taking the study medication. A significantly greater proportion of subjects in the two sumatriptan groups also showed a sustained pain-free response from two to 24 hours after taking study medication as compared to placebo. Significantly more subjects experienced worsening of headache intensity at two and four hours in the placebo group as compared to the active treatment groups. 
Table 3: Number of Patients (\%) Who Sustained A Pain-Free Response Between Two and 24 Hours After Initial Treatment With Study Medication

\begin{tabular}{|c|c|c|c|c|c|}
\hline Population & Placebo & $50 \mathrm{mg}$ & $100 \mathrm{mg}$ & $P$ value & $P$ value \\
\hline & & & & $\begin{array}{l}50 \mathrm{mg} \text { vs } \\
\text { placebo }\end{array}$ & $\begin{array}{l}100 \mathrm{mg} \text { vs } \\
\text { placebo }\end{array}$ \\
\hline ITT & $7(6 \%)$ & $30(24 \%)$ & $34(27 \%)$ & $<0.001$ & $<0.001$ \\
\hline PP & $7(11 \%)$ & $28(29 \%)$ & $32(33 \%)$ & 0.017 & 0.001 \\
\hline
\end{tabular}

The headache recurrence rates observed in our study after initial successful treatment are relatively high when compared to previous sumatriptan clinical trials. ${ }^{20}$ This may be the case because in our study, unlike some previous studies, any resumption of headache however mild within 24 hours was counted as a recurrence, as we were looking at recurrence after a pain-free response. In addition, the fact that our patient population was drawn in large measure from neurology practices or headache clinics, may have resulted in a relatively refractory patient population.

The proportion of subjects pain-free at two hours in this study (where sumatriptan was taken early in the migraine attack within two hours of pain onset and while the pain was still mild) was greater than has been reported in previous clinical trials (in which subjects treated their migraine pain when it was at a moderate or severe level). In our ITT population, $40 \%$ and $50 \%$ of subjects in the sumatriptan $50 \mathrm{mg}$ and sumatriptan $100 \mathrm{mg}$ groups respectively achieved a pain-free state two hours after taking study medication, while only $16 \%$ of placebo subjects were pain-free at two hours. This compares very favorably to the two hour pain-free rates found in clinical trials where subjects were instructed to treat only after the headache had become at least moderate in intensity, and where subjects were allowed to treat up to eight hours after headache onset. In a large metaanalysis of all randomized double blind placebo controlled sumatriptan clinical trials conducted in this way up to that time, it was found that $29 \%$ of subjects treated with sumatriptan 100 $\mathrm{mg}$ and $10 \%$ of subjects in the placebo group were pain-free at two hours. $^{21}$

The two hour pain-free rate of $50 \%$ for sumatriptan $100 \mathrm{mg}$ in our early treatment study is strikingly higher than the $29 \%$ rate found in the meta-analysis of clinical trials ${ }^{21}$ which treated pain at the moderate or severe stage. Although the placebo two hour pain-free responses were also higher in our early treatment trial compared to that found in the meta-analysis (16\% versus $10 \%)$ the placebo-subtracted two hour pain-free rates in our early treatment trial are still substantially higher than in the metaanalysis (34\% cf. 19\%). These results suggest that a greater therapeutic gain is demonstrated when patients initiate sumatriptan therapy at an early stage when their migraine pain is still mild.

A possible physiological explanation for the increased efficacy following early treatment with sumatriptan as compared to treatment later in the attack is the development of central sensitization during migraine headache. During a migraine attack, nociceptive neural activity is transmitted from peripheral sensory nerves to central sensory neurons. It is thought that these central sensory neurons in the trigeminal nucleus caudalis may become sensitized as a result of continuing peripheral nociceptive inputs, a process referred to as central sensitization. ${ }^{22}$ Once central sensitization has occurred during a migraine attack, the patient may develop cutaneous allodynia

Table 4: Number of Patients (\%) With Worsening of Headache (ITT Population)

\begin{tabular}{|c|c|c|c|c|c|}
\hline Population & Placebo & $50 \mathrm{mg}$ & $100 \mathrm{mg}$ & P value & P value \\
\hline & & & & $\begin{array}{l}50 \mathrm{mg} \text { vs } \\
\text { placebo }\end{array}$ & $\begin{array}{l}100 \mathrm{mg} \text { vs } \\
\text { placebo }\end{array}$ \\
\hline 2 hours & $58(53 \%)$ & $41(33 \%)$ & $28(22 \%)$ & 0.002 & $<0.001$ \\
\hline 4 hours & $50(46 \%)$ & $31(25 \%)$ & $17(13 \%)$ & $<0.001$ & $<0.001$ \\
\hline
\end{tabular}


and the attack may become more difficult to treat. As a result, it has been suggested that patients should be encouraged to treat their migraine pain at a mild stage before central sensitization has occurred. Treating before cutaneous allodynia has developed appears to result in greater efficacy of migraine medications as measured by the two hour pain-free end point. ${ }^{23}$

The relatively high pain-free response rates at two hours posttreatment in our study are consistent with the concept that central sensitization may make a migraine attack more resistant to treatment. Our results are also consistent with other studies that have examined the efficacy of triptans when used early in the treatment of migraine attacks. ${ }^{14-17}$ Such studies have also shown relatively high pain-free response rates as compared to others in which the triptan was taken only after the headache had reached a moderate or severe intensity.

Another possibility for the better efficacy of sumatriptan taken early in the migraine attack may be that drug absorption from the gastrointestinal tract may be better early in the attack than later. We think this is unlikely to have been a significant factor as it has been shown that there is no significant difference in sumatriptan pharmacokinetics during a migraine attack as compared to when the same patient is pain-free. ${ }^{24}$

In our study, as compared to previous conventional clinical trials where subjects treated at moderate or severe intensity, the two hour pain-free endpoint was achieved by a higher proportion of subjects not only in the active treatment groups but also by subjects in the placebo group. In the ITT group, $16 \%$ of subjects were pain-free at two hours, compared to $10 \%$ in the metaanalysis of older sumatriptan trials. ${ }^{21}$ This trend towards higher two hour pain-free response rates in early treatment triptan trials is confirmed by other clinical trials. These include a previous early treatment sumatriptan trial where the two hour pain-free placebo response rate was $29 \%$ (14) and a menstrually associated migraine sumatriptan early treatment trial where the placebo response rate was similar. ${ }^{25}$ In a three attack early treatment trial with rizatriptan, the two hour pain-free response rate in the placebo group was $22 \% .{ }^{17}$ In a zolmitriptan early treatment trial, it was $20 \% .^{16}$ In contrast, placebo two hour pain-free rates in traditional trials with rizatriptan and zolmitriptan where patients treated headache attacks at the moderate or severe stage were typically under $10 \% .^{26}$

How placebos relieve migraine headache is not fully understood. Activation of pain modulation systems including endogenous opioid pathways may play a role. ${ }^{27}$ One could speculate that such placebo induced pain modulating mechanisms are more likely to render a migraine attack pain-free at two hours if the placebo is taken very early in the course of the migraine attack. As the pathophysiology of the migraine attack unfolds, a point may be reached quite early in the process where the mechanisms underlying the placebo response are much less likely to terminate the attack. While this difference in the placebo response contributes to the better efficacy of the triptans when taken early in a migraine attack, it is important to point out that the placebo- subtracted response rate (therapeutic gain) is also generally higher in early-treatment triptan clinical trials. Thus the enhanced placebo response cannot account for the higher triptan two hour pain-free response rates. The migraine attack would also appear to be more vulnerable to the therapeutic mechanisms of the triptans in the early stages of its evolution.
One issue in a study such as this in which the patient is requested to treat the headache at a mild stage, is how many of these headaches, if untreated, might have reached a moderate or severe intensity. In most previous triptan studies, head pain of moderate or severe intensity was a prerequisite for treatment. It is important to note that one of our study entry criteria was that the subject typically experienced moderate to severe pain with their migraines. In addition, all subjects completed the Headache Impact Test (HIT-6) which indicated a very severe impact of the migraines for the subjects, with mean scores between 71 and 76 for the three patient groups. Finally, between $97 \%$ and $100 \%$ of subjects in the three groups indicated on the HIT-6 questionnaire that their headaches always, very often, or sometimes reached the severe level. It is therefore very likely that the great majority of headache attacks in our study population would have gone on to at least a moderate intensity. Also supportive of this conclusion is the observation that even by two hours post treatment in the ITT group, $63 \%$ of the 92 patients in the placebo group who had not been rendered pain free by placebo had already progressed to a headache of at least moderate intensity. Nevertheless, it remains possible that subjects who treat at the mild stage may have a higher spontaneous resolution rate of their migraine attacks by two hours than those who treat only once the headache has reached at least moderate intensity.

Our patient population is also somewhat different from those in migraine trials that treated at a moderate or severe pain intensity, as the inclusion criteria for our trial included the presence of an identifiable mild pain phase prior to the subject's headache becoming of greater intensity. However, it might be expected that most migraine sufferers would progress, however briefly, through a mild pain phase as their headache escalates in intensity.

The advantages and disadvantages of early triptan treatment of migraine are clinically relevant. On the one hand, early treatment of a migraine attack at the mild stage and within two hours of pain onset provides a relatively high two hour pain-free rate which reached $57 \%$ in our per protocol analysis. On the other hand, it is generally accepted that if triptans are used on more than ten days a month, patients with migraine are placed at risk for the development of medication overuse headache, with more frequent headache attacks. ${ }^{28}$ Clearly, early triptan treatment could be detrimental if it should lead to escalation of triptan use beyond acceptable limits.

The best approach might be to encourage early triptan use for patients with relatively infrequent migraine attacks, particularly if their attacks are not rendered pain-free at two hours when triptan use is delayed until the pain is moderate or severe. However, for patients with frequent headaches, discretion in triptan use may be advisable, early use being advised for only some of their attacks, particularly if their experience allows them to predict the likely course of any current attack. For other headache attacks, the nature of which is not immediately clear or that occur at less critical times in the patient's day, it may be best to wait and see if the triptan is really needed, using non-specific remedies initially. For any patient with migraine, if triptans are used ten days a month or more, medication use should be carefully reviewed and alternative treatment strategies explored.

Some limitations of this study must be addressed. Firstly, information on whether or not patients were triptan naïve was not 
available. If patients were not triptan naïve, this might have affected the blinding in the trial, and affected the expectations of some of the patients of their study medication. It is possible that if all patients had been triptan naive, the separation between triptan and placebo could have been less. Secondly, in our comparison of therapeutic gain and treatment efficacy between early treatment and later treatment (i.e. when headache pain is moderate or severe), we have based our comparisons on results from previous studies. This would constitute the use of historical controls. Although there are potential flaws with the use of historical controls, we feel this is not a major concern because the historical data came from a meta-analysis.

In conclusion, the treatment of migraine headaches with a triptan early, when the pain is still mild, appears to offer significant benefit to many patients as compared to later treatment. The role of the increased placebo response with early treatment in the observed benefit is intriguing. For patients with frequent migraine attacks, the frequency of triptan use needs to be monitored carefully and the treatment strategy changed if necessary.

\section{ACKNOWLEDGEMENT}

This multicenter clinical trial was funded by GlaxoSmithKline. The investigators who participated in this clinical trial are listed below:

M. Aube, Montreal; N. Bayer, Toronto; W. Becker, Calgary; A. Bellavance, Greenfield Park; G. Boudreau, Laval; C. Bourque, Winnipeg; S. Christie, Ottawa; H. Desai, Windsor: M. Gawel, Toronto; R. Giammarco, Hamilton; K. Giles, Cambridge; A. Goodridge, St. John's; D. Halle, Hull; G. Mackie, Vancouver; S. McKenzie, Mississauga; L. Muresan, Montreal; A. Purdy, Halifax; G. Shapero, Unionville; A. Shuaib, Edmonton; D. Simard, Quebec; L. Sitwell, Ottawa; M. Veilleux, Montreal; C. Voll, Saskatoon; M. Winger, Tecumseh; G. Zlotnik, Montreal; I. Gorfinkel, Toronto.

\section{CONFLICT OF INTEREST}

Please note that F.F. Ahmad, W. Pryse-Phillips and S.D. Simpson are GlaxoSmithKline employees. S. Jelinski is the recipient of the Canadian Headache fellowship for 2004-2005. This fellowship is adjudicated and administered by the Canadian Headache Society, but funded on an annual basis by GlaxoSmithKline. W.J. Becker has held research grants in the past from GlaxoSmithKline and has served as a consultant, but none of these involvements are relevant to this manuscript.

\section{REFERENCES}

1. O'Brien B, Goeree R, Streiner D. Prevalence of migraine headache in Canada: a population-based survey. Int J Epidemiol. 1994; 23:1020-6.

2. Gibbs TS, Fleischer AB, Jr., Feldman SR, et al. Health care utilization in patients with migraine: demographics and patterns of care in the ambulatory setting. Headache. 2003; 43:330-5.

3. Lipton RB, Scher AI, Kolodner K, et al. Migraine in the United States: epidemiology and patterns of health care use. Neurology. 2002; 58:885-94.

4. Gerth WC, Carides GW, Dasbach EJ, et al. The multinational impact of migraine symptoms on healthcare utilisation and work loss. Pharmacoeconomics. 2001; 19:197-206.

5. Fishman P, Black L. Indirect costs of migraine in a managed care population. Cephalalgia. 1999; 19:50-7.
6. Von Korff M, Stewart WF, Simon DJ, Lipton RB. Migraine and reduced work performance: a population-based diary study. Neurology. 1998; 50:1741-5.

7. The Subcutaneous Sumatriptan International Study Group. Treatment of migraine attacks with sumatriptan. N Engl J Med. 1991; 325:316-21.

8. Visser WH, Ferrari MD, Bayliss EM, et al. The Subcutaneous Sumatriptan International Study Group. Treatment of migraine attacks with subcutaneous sumatriptan: first placebo-controlled study. Cephalalgia. 1992; 12:308-13.

9. Nappi G, Sicuteri F, Byrne M, et al. Oral sumatriptan compared with placebo in the acute treatment of migraine. J Neurol. 1994; 241:138-44

10. Cutler N, Mushet GR, Davis R, et al. Oral sumatriptan for the acute treatment of migraine: evaluation of three dosage strengths. Neurology. 1995; 45:S5-9.

11. Sargent J, Kirchner JR, Davis R, Kirkhart B. Oral sumatriptan is effective and well tolerated for the acute treatment of migraine: results of a multicenter study. Neurology. 1995; 45:S10-14.

12. Burstein R, Yamamura H, Malick A, Strassman AM. Chemical stimulation of the intracranial dura induces enhanced responses to facial stimulation in brain stem trigeminal neurons. J Neurophysiol. 1998; 79:964-82.

13. Cady RK, Sheftell F, Lipton RB, et al. Effect of early intervention with sumatriptan on migraine pain: retrospective analyses of data from three clinical trials. Clin Ther. 2000; 22:1035-48.

14. Winner P, Mannix LK, Putnam DG, et al. Pain-free results with sumatriptan taken at the first sign of migraine pain: 2 randomized, double-blind, placebo-controlled studies. Mayo Clin Proc. 2003; 78:1214-22.

15. Scholpp J, Schellenberg R, Moeckesch B, Banik N. Early treatment of a migraine attack while pain is still mild increases the efficacy of sumatriptan. Cephalalgia. 2004; 24:925-33.

16. Klapper J, Lucas C, Rosjo O, Charlesworth B. Benefits of treating highly disabled migraine patients with zolmitriptan while pain is mild. Cephalalgia. 2004; 24:918-24.

17. Mathew NT, Kailasam J, Meadors L. Early treatment of migraine with rizatriptan: a placebo-controlled study. Headache. 2004; 44:669-73.

18. Headache Classification Committee of the International Headache Society. Classification and diagnostic criteria for headache disorders, cranial neuralgias and facial pain. Cephalalgia. 1988; 8 Suppl 7:1-96.

19. Kosinski M, Bayliss MS, Bjorner JB, et al. A six-item short-form survey for measuring headache impact: the HIT-6. Qual Life Res. 2003; 12:963-74.

20. Goadsby PJ. Serotonin 5-HT receptor agonists in migraine. CNS Drugs. 1998; 10:271-86.

21. Ferrari MD, Roon KI, Lipton RB, Goadsby PJ. Oral triptans (serotonin 5-HT(1B/1D) agonists) in acute migraine treatment: a meta-analysis of 53 trials. Lancet. 2001; 358:1668-75.

22. Burstein R. Deconstructing migraine headache into peripheral and central sensitization. Pain. 2001; 89:107-10.

23. Burstein R, Collins B, Jakubowski M. Defeating migraine pain with triptans: a race against the development of cutaneous allodynia. Ann Neurol. 2004; 55:19-26.

24. Sramek JJ, Hussey EK, Blements B, Cutler NR. Oral sumatriptan pharmacokinetics in the migraine state. Clin Drug Invest. 1999; 17:137-44.

25. Nett R, Landy S, Shackelford S, et al. Pain-free efficacy after treatment with sumatriptan in the mild pain phase of menstrually associated migraine. Obstet Gynecol. 2003; 102:835-42.

26. Belsey J. Reconciling effectiveness and tolerability in oral triptan therapy: a quantitative approach to decision making in migraine management. J Clin Res. 2001; 4:105-25.

27. Amanzio M, Benedetti F. Neuropharmacological dissection of placebo analgesia: expectation-activated opioid systems versus conditioning-activated specific subsystems. J Neurosci. 1999; 19:484-94.

28. The International Classification of Headache Disorders: 2nd edition. Cephalalgia. 2004; 24 Suppl 1:9-160. 